\title{
„Die Beteiligung der Legislative bei Vorbehalten zu und Kündigung von völkerrechtlichen Verträgen“"
}

Dissertation vorgelegt von Juliane Hettche

Erstgutachter: Prof. Dr. Bernd Grzeszick

Zweitgutachter: Prof. Dr. Hanno Kube

Institut für Staatsrecht, Verfassungslehre und Rechtsphilosophie 


\section{A. Darstellung des Themas}

In der vorgelegten Arbeit wird versucht, eine systemgerechte und zeitgemäße Antwort auf die Frage zu geben, ob und welche Beteiligungsrechte der Legislative, also Bundestag und Bundesrat, bei Vorbehalten zu und Kündigung von völkerrechtlichen Verträgen nach dem Grundgesetz (GG) zustehen.

Da Art. 59 Abs. 2 S. 1 GG zu dieser Problematik keine expliziten Vorgaben enthält, ist zu untersuchen, ob zur Herleitung solcher Beteiligungsrechte auf Grundsätze zurückgegriffen werden kann, die auch die Rolle der Legislative im Innenverhältnis bestimmen. Dagegen richtet sich allerdings das BVerfG, das trotz steigender Bedeutung von völkerrechtlichen Verträgen die Beteiligungsmöglichkeiten der Legislative bei Art. 59 Abs. 2 S. 1 GG als Sonderkompetenz im Bereich exekutivischer Eigenverantwortung restriktiv auslegt.

Die Relevanz des Themas ergibt sich daraus, dass die Annahme von Beteiligungsrechten der Legislative bei Vorbehalten zu oder der Kündigung von völkerrechtlichen Verträgen eine Umgehungsgefahr von deren im Zustimmungsgesetz erklärten Willen durch die Exekutive mindert. Zudem kann die eigeninitiative Einlegung von Vorbehalten zu oder der Kündigung von einem völkerrechtlichen Vertrag die Entscheidungsfreiheit der Legislative wahren und möglicherweise eine Alternative zum Treaty Override darstellen. Die verstärkte Umstrittenheit von Themen im Bereich völkerrechtlicher Verträge und in der Außenpolitik zwischen Regierung und Mehrheitsfraktion erhöht ebenfalls die Relevanz des Themas.

\section{B. Wesentliche Ergebnisse der Arbeit}

Eine Lösung auf die Frage, ob und wie die Legislative bei Vorbehalten zu und Kündigung von völkerrechtlichen Verträgen zu beteiligen ist, wird durch Auslegung des Art. 59 Abs. 2 S. 1 GG gesucht. Dabei wird auf die klassisch-hermeneutische Auslegungsmethode zurückgegriffen in der Weise, dass eine Auslegung zunächst unter Beachtung des subjektiven Willens des Gesetzgebers betrieben wird und nur sofern dies nicht weiter führt, der objektive Ansatz beachtet werden soll.

\section{Auslegung des Wortlauts des Art. 59 Abs. 2 S. 1 GG}

Art. 59 Abs. 2 S. 1 GG sagt explizit nichts zur Existenz von Beteiligungsrechten der Legislative bei einseitigen Handlungen wie Vorbehalt und Kündigung im Rahmen völkerrechtlicher Verträge. Teilweise wird er sehr restriktiv nur auf den Abschluss völkerrechtlicher Verträge bezogen, obwohl er, verstanden als Verweis auf alle Handlungen im Umfeld von Verträgen, eine weitere Auslegung zugunsten solcher Beteiligungsrechte durchaus zulässt. Da die „Zustimmung“ der gesetzgebenden Körperschaften in Form eines „Bundesgesetzes“ gefordert wird, wobei letztere Formulierung den Verweis auf die üblichen Initiativrechte in Art. 76 GG nahelegt, kann Art. 59 Abs. 2 S. 1 GG zugunsten der Beteiligung der Legislative sowohl über Zustimmungs- als auch über Initiativrechte ausgelegt werden. $\mathrm{Ob}$ die fehlende explizite Erwähnung der Initiativrechte in Art. 59 Abs. 2 S. 1 GG als „,beredtes Schweigen“ zu deuten ist, das solche eben ausschließt, kann nur anhand der übrigen Auslegungsmethoden geklärt werden.

\section{Historische Auslegung des Art. 59 Abs. 2 S. 1 GG}

Die Betrachtung der Gesetzgebungsmaterialien zu Art. 59 Abs. 2 S. 1 GG gibt keinen Aufschluss darüber, ob der Parlamentarische Rat als historischer Gesetzgeber Beteiligungsmöglichkeiten der Legislative bei einseitigen Akten vorsehen wollte oder nicht. Bei Vorgängernormen des Art. 59 Abs. 2 S. 1 GG in der RV und der WRV wurden solche 
Beteiligungsrechte lediglich bei der Kündigung diskutiert und durch die herrschende Meinung verneint. Es ist allerdings nicht klar, ob der Parlamentarische Rat diese Auslegung für das GG so übernehmen wollte. Gegen die Übernahme dieser Auslegung spricht, dass sie schon unter der RV der Kritik ausgesetzt war. Unter der WRV entsprach sie zudem nicht der Systematik der sonstigen Verfassung: Die ihr zugrunde liegende Sichtweise der Exekutive als mächtiges Zentrum auswärtiger Gewalt mochte unter der monarchisch geprägten RV noch passend gewesen sein, unter der parlamentarisch-demokratischen WRV war sie es jedenfalls nicht mehr. Betrachtet man die rechtsinterne Entwicklung der Vorgängernormen des Art. 59 Abs. 2 S. 1 GG, so wird deutlich, dass selbst im Kaiserreich der Monarch nicht unbestritten alleiniger Inhaber der auswärtigen Gewalt war und von der RV bis zum GG die Stellung der Legislative gegenüber der Exekutive in diesem Bereich immer weiter gestärkt worden ist. Gerade diese Tendenz zur Parlamentarisierung im auswärtigen Bereich steht im Gegensatz zur fehlenden Zulässigkeit der Beteiligung der Legislative jedenfalls bei der Kündigung völkerrechtlicher Verträge im Rahmen der RV und der WRV und schließlich auch unter dem GG.

Konkrete Vorgaben zur Beteiligung der Legislative bei Vorbehalt und Kündigung völkerrechtlicher Verträge liefert die historische Auslegung insofern nicht. Sie lässt allerdings für den systematischen Teil der Arbeit bedeutsame Schlussfolgerungen zu: Einerseits ist Vorsicht dabei geboten, Schlussfolgerungen zur RV und monarchisch geprägten Staatskonzeptionen auf das Gewaltenteilungssystem des GG zu übertragen, da heute nicht mehr die demokratisch legitimierte Legislative der monarchischen Exekutive gegenüber steht, sondern alle Gewalten demokratisch legitimiert sind. Zudem wird eine besondere Bedeutung der gesetzgeberischen Tätigkeit durch die Art. 81 Abs. 2, 41 Abs. 1, 101, 102 Abs. 2 S. 1 HChE in der Fortentwicklung durch den Parlamentarischen Rat nahegelegt, die sich auch bei der vertikalen und horizontalen Kompetenzverteilung im auswärtigen Bereich ausdrückt. Dem entspricht auch der aus den Diskussionen des Parlamentarischen Rates hervorgehende Zweck des Art. 59 Abs. 2 S. 1 GG: Das Zustimmungserfordernis der Legislative vor Ratifikation des Vertrags soll deren Entscheidungsfreiheit wahren und den Gleichlauf von Staats- und Völkerrecht garantieren.

\section{Systematische Auslegung des Art. 59 Abs. 2 S. 1 GG: Übertragung von Grundsätzen des Innenverhältnisses auf das Außenverhältnis}

Die systematische Auslegung des Art. 59 Abs. 2 S. 1 GG erfolgt vor der Annahme, dass dieser zwar als deutlichste Entscheidung des Verfassungsgesetzgebers in Bezug auf Beteiligungsrechte der Legislative $\mathrm{zu}$ völkerrechtlichen Verträgen Ansatzpunkt der Auslegung sein muss, allerdings die tragenden Prinzipien des GG bei offenen Fragen als Interpretationskriterien Orientierung bieten können. Bei der Auslegung des Art. 59 Abs. 2 S. 1 GG wird angenommen, dass Grundzüge und Verfahrensweisen, die die Rolle der Legislative im Innenverhältnis bestimmen, auch auf das Außenverhältnis übertragen werden und insofern der Herleitung von Beteiligungsrechten bei Vorbehalt und Kündigung dienen können.

\section{Die Rolle der Legislative im Innenverhältnis prägende Grundsätze}

Die Rolle der Legislative im Innenverhältnis wird bestimmt durch die unmittelbare demokratische Legitimation des Parlaments, die dieses wegen der aus dem Demokratieprinzip und der Volkssouveränität hergeleiteten Notwendigkeit des unmittelbaren Einflusses des Volks auf jegliche Verwirklichung der Staatsgewalt zum unentbehrlichen Organ sowohl im Rahmen der organisatorisch-personellen als auch der sachlich-inhaltlichen demokratischen Legitimation macht. Deshalb allerdings von einer besonders exponierten Stellung des Parlaments gegenüber der Bundesregierung auszugehen ist nicht gerechtfertigt, da diese zum einen wegen des Charakters der Bundestagswahl als Personenwahl des Kanzlers und der 
Annäherung des Regierungsamts zum Abgeordnetenmandat über eine ganz ähnliche demokratische Legitimation verfügt, zum anderen das GG auch die mittelbare demokratische Legitimation nicht unbedingt als geringwertiger als die unmittelbare demokratische Legitimation begreift.

Massiv wird die Rolle der Legislative im Innern zudem geprägt durch die Bedeutung des Gesetzes als Handlungsinstrument: Der Vorrang des Gesetzes, der als Mittel der sachlichdemokratischen Legitimation die Bindung der anderen Gewalten an den in Gesetzesform geronnenen Willen des Gesetzgebers erlaubt, ist dabei geeignet, der Legislative gegenüber diesen eine hervorgehobene Stellung $\mathrm{zu}$ verschaffen. Ist die Legislative einmal tätig geworden, setzt der Diskontinuitätsgrundsatz voraus, dass sie im Sinne des Demokratieprinzips jede gesetzgeberische Entscheidung revidieren kann. Sofern die Handlungsform des Gesetzes vorgeschrieben oder gewählt wird, wird über die ggfs. notwendige Beteiligung des Bundesrates dann auch der Föderalismusgedanke verwirklicht.

Zwingend tätig werden muss die Legislative im Innenverhältnis bei „wesentlichen“ Entscheidungen in grundlegenden normativen Bereichen, insbesondere im Bereich der Grundrechtsausübung. Vor allem die transparente Verfahrensweise bei der Schaffung eines Parlamentsgesetzes mit öffentlicher Debatte unter Beteiligung der Opposition soll für diese Schlussfolgerung sprechen. Dieser auch vom BVerfG vertretenen Wesentlichkeitstheorie wird zwar zu Recht der Vorwurf der Vagheit gemacht in Bezug auf die Bestimmung dessen, was wesentlich ist. Trotzdem überzeugt sie mehr als alternative Ansätze, wie in der Arbeit detailliert dargelegt wird.

Das Verhältnis der Legislative zur Exekutive im Innenbereich wird bestimmt durch verschiedene Funktionen der Gewaltenteilung: Gerade wegen der Beteiligung der Opposition im parlamentarischen Verfahren, allerdings auch wegen eines bestehenden Spannungsverhältnisses der Parlamentsmehrheit gegenüber der Regierung ist von einer aus dem Gewaltenteilungsprinzip resultierenden kontrollierenden Funktion der Legislative gegenüber der Exekutive auszugehen. Zudem erfolgt die Abgrenzung des Aufgabenbereichs der Legislative gegenüber der Exekutive nach der Funktionenlehre des BVerfG über die Wesentlichkeitstheorie, was zur Etablierung eines Kernbereichs der Legislative führt, soweit eben der allgemeine Gesetzesvorbehalt reicht. Darüber hinaus soll die Legislative nach der Funktionenlehre entsprechend ihrer Eignung in Bezug auf Zusammensetzung, Funktion und Verfahrensweise im Einzelfall aus ,guten Gründen“ handeln können, die schon in Effizienzvorteilen, aber auch in Gründen aus dem Bereich des Rechtsstaats- und Demokratieprinzips bestehen können. Verschlossen soll ihr lediglich der Bereich bleiben, der unter der Kernbereichslehre als „Kernbereich der Exekutive“ definiert ist. Die Anwendbarkeit der Kernbereichslehre wird in der Arbeit kritisch diskutiert und es werden alternative Konzepte dargestellt, die weniger strikte Grenzen für Zugriffsrechte der Gewalten untereinander ziehen.

\section{Ansichten in Literatur und Rechtsprechung}

\section{a) BVerfG und ihm folgende Literatur: Keine Übertragung der im Innenverhältnis geltenden Grundsätze auf das Außenverhältnis}

Geht man zur Betrachtung der Rolle der Legislative im Außenverhältnis, insbesondere im Bereich des Art. 59 Abs. 2 S. 1 GG über, so wird deutlich, dass jedenfalls das BVerfG und die ihm folgende Literaturansicht nicht von einer Übertragung der Grundsätze und Verfahrensweisen des Innen- auf das Außenverhältnis ausgehen. Anknüpfend an die Funktionenlehre sieht das BVerfG den Bereich auswärtiger Gewalt als Domäne und Kernbereich der Exekutive, in dem Art. 59 Abs. 2 S. 1 GG eine nicht erweiternd auszulegende 
Sonderkompetenz der Legislative darstellen soll und löst den Organkompetenzkonflikt insofern über das Gewaltenteilungsprinzip. Die Tätigkeit der Legislative durch das Zustimmungsgesetz wird nicht als Gesetzgebung, sondern als Regierungsakt in Gesetzesform definiert, bei dem gerade nicht die üblichen Initiativ-, Gestaltungs- oder Kontrollbefugnisse der Legislative Anwendung finden sollen. Eine Beteiligung der Legislative über ein Zustimmungsgesetz soll dabei lediglich bei Abschluss oder (konkludenter) Änderung von Verträgen stattfinden, im letzteren Fall vor allem dann, wenn der Ermächtigungsrahmen des ursprünglichen Vertrags durch die Exekutive überschritten wurde.

In gewissem Gegensatz zu dieser Rechtsprechung stehen einerseits die Urteile des BVerfG zur Verbandskompetenz, in denen es um die Erhöhung demokratischer Legitimation auf Ebene supra- oder internationaler Organisationen oder Absprachen geht, andererseits auch die Urteile zum Einsatz der Bundeswehr im Ausland. In beiden Rechtsprechungslinien werden die Rechte der Legislative bzw. des Parlaments gestärkt - vor allem wird die Bedeutsamkeit der öffentlichen Diskussion unter Beteiligung der Opposition und der Rückbindung einer Entscheidung an den Volkssouverän deutlich gemacht. Ebenso wird in diesen Entscheidungen das Parlament im Gegensatz zu den Urteilen zum Organkompetenzkonflikt als fähig sowohl zu schnellem als auch zu geheimen Handeln gehalten.

\section{b) Gegensätzliche Literaturansicht: Erhebliche Mitspracherechte der Legislative im Außenverhältnis}

Die Literatur hält der Ansicht des BVerfG entgegen, dass das GG im Bereich auswärtiger Gewalt erhebliche Mitspracherechte des Parlaments festlegen würde und argumentiert für die Annahme einer „Staatsleitung zur gesamten Hand“ im Bereich auswärtiger Gewalt mit Exekutive und Legislative als zwei Funktionsträgern. Für diese Annahme wird auch die Notwendigkeit des öffentlichen Verfahrens der Legislative gerade bei Verrechtlichung des auswärtigen Bereichs durch völkerrechtliche Verträge angeführt. Als Folge der geteilten Kompetenz im Bereich auswärtiger Gewalt sieht diese Literaturansicht auch die erweiternde Auslegung des Art. 59 Abs. 2 S. 1 GG als möglich an.

\section{Lösung durch Übertragung und Abwägung der im Innenverhältnis geltenden Grundsätze und Verfahrensweisen}

Die zuletzt beschriebene Literaturansicht bleibt allerdings zu abstrakt, um das „ob“ und „wie“ von Beteiligungsrechten der Legislative im Bereich von Vorbehalten zu und Kündigung von völkerrechtlichen Verträgen festzulegen. Konkretere Vorgaben lassen sich herleiten aus den auch im Innenverhältnis geltenden Grundsätzen und Verfahrensweisen, auf die letztlich von der genannten Literaturansicht verwiesen wird und auf die Art. 59 Abs. 2 S. 1 GG seinem Zweck entsprechend auch aufbaut: Die Wesentlichkeitstheorie, der Vorrang des Gesetzes, der Diskontinuitätsgrundsatz, das Initiativrecht aus Art. 76 Abs. 1 GG, der Föderalismusgedanke und zuletzt das Gewaltenteilungsprinzip. Angeknüpft werden kann dabei an den Gedanken, dass wegen der verstärkten Regulierungstendenzen im auswärtigen Bereich dieser nicht mehr als unnormierbar gelten kann und insofern ein Gleichlauf der anwendbaren Prinzipien und Verfahrensweisen im Innen- und Außenverhältnis stattfinden muss.

\section{a) Grundrechtsbindung der Legislative im Außenverhältnis}

Gewissermaßen als Vorfrage der Anwendung der Wesentlichkeitstheorie im Außenverhältnis ist zunächst die Grundrechtsgeltung im Bereich völkerrechtlicher Verträge herzuleiten. Hier sind sich das BVerfG und die Literatur darin einig, dass die deutsche Staatsgewalt bei Abschluss völkerrechtlicher Verträge der Grundrechtsbindung unterliegt und Art. 1 Abs. 3 GG sowohl die Handlungen der Exekutive als auch der Legislative in diesem Bereich 
bestimmt. Lediglich beim Umfang des Grundrechtsschutzes können durch Schutzbereichsbeschränkungen oder Schrankenregelungen wegen der Eigenart der Außenbeziehungen gegenüber dem Innenverhältnis Kompromisse gemacht werden. Die Legislative wird bei völkerrechtlichen Verträgen durch die Grundrechtsbindung kaum einer Handlungspflicht unterliegen - allerdings kann sie durch Beteiligungsrechte bei Vorbehalt und Kündigung unter Umständen umfassenderen Grundrechtsschutz leisten.

\section{b) Anwendung der Wesentlichkeitstheorie im Außenverhältnis}

Die Grundrechtsbindung der Legislative bei völkerrechtlichen Verträgen spricht schon für die Anwendung der Wesentlichkeitstheorie, die oftmals bei „Grundrechtsrelevanz" einer Maßnahme greift. Für die Anwendung der Wesentlichkeitstheorie spricht zudem der Verweis des Art. 59 Abs. 2 S. 1 Var. 2 GG auf den im Innern geltenden Gesetzesvorbehalt. Um dem Zweck der Sicherung der Entscheidungsfreiheit der Legislative und des Gleichlaufs von Staats- und Völkerrecht gerecht zu werden, müssen zwingende Beteiligung der Legislative im Innenverhältnis und bei völkerrechtlichen Verträgen parallel verlaufen. Im Bereich des Art. 59 Abs. 2 S. 1 Var. 2 GG ist die Beteiligung der Legislative insofern bloß als Vorverlagerung des innerstaatlichen Verfahrens aus Effektivitätsgründen zur Wahrung der völkerrechtlichen Verpflichtung zu sehen. Der Anwendung der Wesentlichkeitstheorie auch im Bereich des Art. 59 Abs. 2 S. 1 Var. 2 GG steht dabei auch nicht das Argument entgegen, die Variante des politischen Vertrags würde infolge dieser weiten Auslegung des Begriffs des gesetzesinhaltlichen Vertrags entleert: Einerseits deutet ein solcher Effekt höchstens auf eine $\mathrm{zu}$ weite Auslegung des Gesetzesvorbehalts im Innern hin. Andererseits hat der Parlamentarische Rat die Variante des politischen Vertrags eingefügt, weil er befürchtete, dass die notwendige Beteiligung der Legislative unter der Variante der gesetzesinhaltlichen Verträge nicht erfolgen würde. Der politische Vertrag kann deshalb als kodifizierter Fall der Wesentlichkeitstheorie begriffen werden, dessen Fassung unter Art. 59 Abs. 2 S. 1 Var. 2 GG nicht schadet.

Der Verweis auf die Wesentlichkeitstheorie in Art. 59 Abs. 2 S. 1 Var. 2 GG ist als Ausdruck eines allgemeinen Prinzips zu verstehen, das zur Vermeidung der Umgehung des Zwecks des Art. 59 Abs. 2 S. 1 GG eine Beteiligung der Legislative bei allen wesentlichen Entscheidungen im Bereich völkerrechtlicher Verträge, auch bei Vorbehalt und Kündigung, fordert.

\section{c) Anwendung der für ein Gesetz relevanten Grundsätze und Verfahrensweisen}

Entgegen der Ansicht des BVerfG ist mangels anderer Andeutung im Wortlaut des Art. 59 Abs. 2 S. 1 GG und in der Systematik des GG und der GOBT davon auszugehen, dass das Zustimmungsgesetz ein „Gesetz“ im herkömmlichen Sinne darstellt, auf das die üblichen Grundsätze und Verfahrensweisen Anwendung finden. Schon diese Annahme spricht für die Bejahung der üblichen Initiativrechte der Legislative nach Art. 76 Abs. 1 GG im Bereich des Zustimmungsgesetzes. Ebenfalls für diese Sichtweise angeführt werden kann die Annahme einer bloßen Vorverlagerung des Gesetzgebungsverfahrens beim Zustimmungsgesetz zu gesetzesinhaltlichen Verträgen und die Tatsache, dass Art. 59 Abs. 2 S. 1 GG anders als Art. 110 Abs. 3, 115 a Abs. 1 GG ein Initiativrecht der Legislative nicht ausschließt.

Die Anwendung des Diskontinuitätsgrundsatzes im Bereich völkerrechtlicher Verträge wird sogar vom BVerfG bejaht: Gegen seine Geltung kann nur argumentieren wer das Zustimmungsgesetz aus Gründen der Völkerrechtsfreundlichkeit als „Sperre“ gegenüber der Ausübung der gesetzgeberischen Gestaltungsmöglichkeiten begreift. Doch selbst nach dieser Ansicht sollte der Diskontinuitätsgrundsatz jedenfalls insoweit Anwendung finden, als der Gesetzgeber durch Initiierung eines Vorbehalts $\mathrm{zu}$ oder der Kündigung von einem 
völkerrechtlichen Vertrag das Zustimmungsgesetz mit Außenwirkung in völkerrechtskonformer Weise ändern kann. Die Änderung durch Vorbehalt sollte lediglich bei erfolgter Übertragung von Hoheitsrechten versperrt sein. Initiierung und Beschluss einer Kündigung sollte dem Gesetzgeber auch in dieser Konstellation möglich sein, um ihm mit der Rückholung der Hoheitsrechte die Änderung des Zustimmungsgesetzes zu erlauben.

Ebenso kann die Geltung des Vorrangs des Gesetzes auf das Außenverhältnis übertragen werden: Obwohl das BVerfG die Geltung der üblichen Initiativ-, Gestaltungs- und Kontrollbefugnisse der Legislative in Bezug auf das Zustimmungsgesetz verneint, sieht es das Zustimmungsgesetz doch als inhaltlich gegenüber der Exekutive verbindlich an und verbietet ultra vires Akte. Das Zustimmungsgesetz ist dabei allerdings so auszulegen, dass es die Exekutive nicht nur zum Abschluss des Vertrags, sondern konkludent auch zum Unterlassen der Ratifikation im Fall von unvorhergesehenen Entwicklungen ermächtigt. Ebenfalls im Außenverhältnis findet schon wegen der Anordnung der Mitwirkung des Bundesrates nach Art. 59 Abs. 2 S. 1 GG der Föderalismusgedanke Anwendung, dem vermehrte Zustimmungsund Initiativrechte der Legislative und damit auch des Bundesrates zugutekommen.

\section{d) Anwendung des Gewaltenteilungsprinzips im Außenverhältnis}

Letztlich bestimmt auch das Gewaltenteilungsprinzip das Handeln der Legislative und ihr Verhältnis zur Exekutive im Außenverhältnis. Soweit nicht die Kernbereichslehre ohnehin wegen ihrer Unklarheit und Unvereinbarkeit mit einem gewaltenverschränkenden System abzulehnen ist, ist jedenfalls davon auszugehen, dass im Bereich völkerrechtlicher Verträge, in dem Art. 59 Abs. 2 S. 1 GG eine Mitwirkung der Legislative anordnet, gerade kein Kernbereich der Exekutive besteht. Bei Anwendung der Funktionenlehre oder alternativer Argumentationsstrukturen spricht jedenfalls die Schaffung einer abstrakten und generellen Regelung, wie sie durch völkerrechtliche Verträge und auch durch Vorbehalt und Kündigung getroffen wird, für ein Tätigwerden der Legislative. Nach den neueren Urteilen des BVerfG zur Funktionenlehre sollten auch aus Rechtsstaats- und Demokratieprinzip resultierende Figuren wie die Wesentlichkeitstheorie, das Diskontinuitätsprinzip oder der Vorrang des Gesetzes für deren Handlungsbefugnis sprechen. Ein Handeln der Exekutive ist nach den gleichen Ansätzen dort zu bejahen, wo persönliche Beziehungen gepflegt werden, es wegen Erfordernis gegenseitiger Kompromisse mit einer nicht an das GG gebundenen Partei um einen wenig normierbaren Bereich geht oder Entscheidungen mit Einzelfallcharakter getroffen werden müssen. Nach diesen Kriterien ist die Exekutive vor allem für eine funktionsfähige Außenvertretung der BRD zuständig. Die Notwendigkeit von schnellem und geheimen Handeln wird nicht als Argument für ein alleiniges Tätigwerden der Exekutive gesehen, da dazu selbst nach Ansicht des BVerfG heute auch die Legislative fähig sein soll. Sofern eine Beteiligung der Legislative als uneffektiv angesehen wird, ist dies mit den Argumenten zu akzeptieren, dass Art. 59 Abs. 2 S. 1 GG im Grundsatz diese Uneffizienz schon vorgibt und im Übrigen das Gewaltenteilungsprinzip gerade nicht der Effizienz an sich, sondern vielmehr der Selbstbestimmung des Einzelnen diente, die auch durch die Beteiligung der Legislative gerade geschützt werden kann. Aufgrund der im Grundsatz schon vorgesehenen Beteiligung der Legislative in Art. 59 Abs. 2 S. 1 GG wird auch davon ausgegangen, dass der Exekutive im Bereich der Außenvertretung nicht durchgängig die vollständige Entscheidungsfreiheit gewährleistet sein muss, ob überhaupt ein völkerrechtliches Rechtsgeschäft vorzunehmen ist.

\section{Abwägungsvorgang und Ergebnisse}

Die Abwägung der genannten Grundsätze und Verfahrensweisen in der jeweiligen Konstellation führt $\mathrm{zu}$ der Festlegung, ob und welches Beteiligungsrecht der Legislative 
zulässig ist. Insofern besteht die Grundannahme, dass die Legislative bei Vorbehalt und Kündigung beteiligt werden kann. Keines der Kriterien gilt dabei als unabwägbar, wobei bezüglich der Grundrechtsbindung eine Einschränkung nur durch Schutzbereichsbeschränkung oder Schrankenregelung des Grundrechts stattfinden kann. Durch den beschriebenen Abwägungsmechanismus gelangt man zu folgenden Ergebnissen:

\section{a) Beteiligung der Legislative bei Vorbehalten zu völkerrechtlichen Verträgen}

Aufgrund der Argumente vor allem aus dem Bereich der Wesentlichkeitstheorie ergibt sich, dass die Legislative der Einlegung eines Vorbehalts vor Ratifikation durch die Exekutive grundsätzlich zustimmen muss. Auf diese Zustimmung kann nach der Funktionenlehre verzichtet werden, wenn deren Einholung der Exekutive zeitlich unmöglich ist. Ebenso unterstützt die Wesentlichkeitstheorie die Argumentation für eine Zustimmung der Legislative zur Einlegung eines Vorbehalts nach Ratifikation, weil ein solcher Vorbehalt die Rechtslage im Innern verändert. Das Gewaltenteilungsprinzip führt hier nicht zu Einschränkungen.

Nach der Wesentlichkeitstheorie ist auch eine Zustimmung der Legislative zur Nichtausübung oder Rücknahme eines Vorbehalts nötig, weil diese Handlung im Innern Veränderungen auslösen würde, die eines Gesetzes bedürften. Auch aus dem Vorrang des Gesetzes kann für ein solches Zustimmungsrecht argumentiert werden, weil der Ermächtigungsrahmen des den Vorbehalt beinhaltenden Zustimmungsgesetzes verlassen werden würde. Das Gewaltenteilungsprinzip steht dem Zustimmungsrecht hier nicht entgegen.

Aus der Abwägung von Wesentlichkeitstheorie, dem Vorrang des Gesetzes und dem Gewaltenteilungsprinzip ergibt sich, dass es der Legislative möglich sein muss, im Zustimmungsgesetz Vorgaben für Reaktionen der Exekutive auf Vorbehalte fremder Staaten zu machen. Sofern sie dies unterlässt, gilt die Exekutive als zur Vornahme aller Reaktionsmöglichkeiten ermächtigt. Bei der Ablehnung eines Vorbehalts durch den Vertragspartner lässt sich hingegen kein Zustimmungsrecht der Legislative konstruieren, da bei der Ablehnung nicht die deutsche Exekutive handelt und der Einlegung des Vorbehalts durch die Legislative ohnehin schon zugestimmt wurde.

Aus der Anwendung des Art. 76 Abs. 1 GG auf das Zustimmungsgesetz lässt sich herleiten, dass der Legislative dort, wo sie einem Vertrag gemäß Art. 59 Abs. 2 S. 1 GG zustimmen muss, auch ein Initiativrecht für ein solches Zustimmungsgesetz zuzugestehen ist, sofern ihr Initiativrecht nicht schon im Innern ausgeschlossen ist. Sie kann deshalb die Zustimmung auch mit der Maßgabe erteilen, dass die Exekutive einen Vorbehalt erklären soll. Das Gewaltenteilungsprinzip steht dem nicht entgegen.

Das Diskontinuitätsprinzip unterstützt die Annahme, dass ein Initiativrecht der Legislative für Vorbehalte auch nach Ratifikation des Vertrags vorliegen muss. Da hier innerhalb des Art. 59 Abs. 2 S. 1 GG ein Spannungsverhältnis zwischen Gewaltenteilungsprinzip, Entscheidungsfreiheit der Legislative und Gleichlauf von Staats- und Völkerrecht zugunsten der beiden letzten als Ausformung von Demokratie- und Rechtsstaatsprinzip aufzulösen ist, steht das Gewaltenteilungsprinzip einem solchen Initiativrecht nicht entgegen. Aufgrund dieser Argumentation kann das Gewaltenteilungsprinzip auch einem auf den Diskontinuitätsgrundsatz gestützten Initiativrecht der Legislative zur Rücknahme eines Vorbehalts nicht entgegengehalten werden.

Trotz ihres Zustimmungsrechts zur Einlegung eines Vorbehalts ist ein Initiativrecht der Legislative, die Exekutive zum Verzicht auf einen Vorbehalt zu zwingen, nach der Wesentlichkeitstheorie zu bejahen. Dies soll der Legislative einen Einfluss auch in den 
Situationen $\mathrm{zu}$ bewahren, in denen die Exekutive bei Eilbedürftigkeit kein Zustimmungsverfahren der Legislative mehr durchführen kann.

\section{b) Beteiligung der Legislative bei Kündigung von völkerrechtlichen Verträgen}

Sofern ein völkerrechtlicher Vertrag nicht explizit ein Kündigungsrecht enthält, dem die Legislative bereits vor Vertragsschluss zugestimmt hat, muss die Legislative der Kündigung völkerrechtlicher Verträge nach Art. 59 Abs. 2 S. 1 GG zustimmen. Dieses Zustimmungsrecht lässt sich aus dem Vorrang des Gesetzes und aus der Wesentlichkeitstheorie ableiten und wird durch die Grundrechtsgeltung im Außenverhältnis unterstützt.

Aus dem Diskontinuitätsgrundsatz lässt sich ein Initiativrecht der Legislative zur Kündigung völkerrechtlicher Verträge herleiten. Das Gewaltenteilungsprinzip unterliegt in der Abwägung der Wahrung der Entscheidungsfreiheit der Legislative und dem Gleichlauf von Staats- und Völkerrecht und steht insofern dem Initiativrecht nicht entgegen. Ein Initiativrecht der Legislative, eine Kündigung zu verhindern, ist wegen des ausreichenden Schutzes der Legislative durch das Zustimmungserfordernis zu dieser Kündigung abzulehnen.

\section{c) Art der Beteiligung}

Obwohl teilweise eine Ausübung der Beteiligungsrechte der Legislative durch schlichten Parlamentsbeschluss vorgeschlagen wird, ist außer in Fällen besonderer Eilbedürftigkeit oder Geheimhaltung im Ergebnis eine Beteiligung über förmliches Gesetz nötig. Dafür spricht die Herleitung der Zustimmungsrechte aus Art. 59 Abs. 2 S. 1 GG, insbesondere bei der Argumentation aus der Wesentlichkeitstheorie und dem Vorrang des Gesetzes sowie die dann auch erfolgende, dem Föderalismusgedanken entsprechende Beteiligung des Bundesrates. Initiativrechte können teilweise sowieso nur über das Zustimmungsgesetz ausgeübt werden; teilweise spricht für ihre bindende Ausübung durch Beschluss eines Gesetzes die Anknüpfung an die Gesetzgebungstätigkeit im Innern und an den Zweck des Art. 59 Abs. 2 S. 1 GG.

\section{Teleologische Auslegung des Art. 59 Abs. 2 S. 1 GG}

Im Rahmen der teleologischen Auslegung wird zunächst der historisch festgestellte Zweck, der die Ergebnisse der systematischen Auslegung stützt, als eine Art „kleinster gemeinsamer Nenner" der subjektiven und objektiven Auslegungsmethode akzeptiert. Bei der Ermittlung eines darüber hinausgehenden, zeitgemäßen Zwecks des Art. 59 Abs. 2 S. 1 GG wird festgestellt, dass jedenfalls der Zweck der Schaffung einer stärkeren demokratischen Legitimation auf Ebene internationaler Organisationen keine stärkere Beteiligung der Legislative bei Vorbehalt und Kündigung rechtfertigen würde, da diese Instrumente Legitimation gerade zurücknehmen und nicht erhöhen. Im Übrigen bestehen Zweifel, ob sich ein solcher Zweck überhaupt in Art. 59 Abs. 2 S. 1 GG hineinlesen lässt oder ob dazu nicht eine neue Regelung geschaffen werden müsste.

Entsprechend der objektiven Methode und eines modernen Ansatzes der subjektiven Methode kann Art. 59 Abs. 2 S. 1 GG in Anbetracht der verstärkten Internationalisierung und grenzüberschreitenden Zusammenarbeit so ausgelegt werden, dass er nicht nur die Entscheidungsfreiheit der Legislative gegenüber der nationalen Exekutive, sondern auch gegenüber internationalen Organisationen und bei der Durchführung völkerrechtlicher Verträge sichern soll. Dies rechtfertigt zusätzlich zu den systematischen Argumenten ein die Exekutive bindendes Initiativrecht zur Einlegung von Vorbehalten zu und Erklärung von Kündigungen von völkerrechtlichen Verträgen. 